\title{
Rapid aneurysmal degeneration of a Stanford type B aortic dissection in a patient with Loeys-Dietz syndrome
}

\author{
Richard S. Lee, MD, ${ }^{a}$ Shafie Fazel, MD, ${ }^{a}$ Ulrike Schwarze, MD, ${ }^{d}$ Dominik Fleischmann, MD, ${ }^{b}$ Gerald J. Berry, MD, ${ }^{e}$
}

David Liang, MD, ${ }^{\mathrm{D}}$ D. Craig Miller, MD, ${ }^{\mathrm{a}}$ and R. Scott Mitchell, MD, ${ }^{a}$ Stanford, Calif, and Seattle, Wash

$\checkmark$ Supplemental material is available online. the case of a 23-year-old woman whose condition satisfied the clinical criteria for LDS and who carried a mutation in the $T G F B R 2$ gene, presenting with a rapidly expanding acute type B aortic dissection.

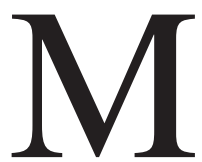

utations in the genes encoding transforming growth factor $\beta$ (TGF $\beta$ ) receptors 1 and 2 (TGFBR1, TGFBR2) have recently been associated with an autosomal dominant syndrome of aortic aneurysms and abnormal craniofacial, neurocognitive, and skeletal development (Loeys-Dietz syndrome, LDS). ${ }^{1}$ Clinical evidence suggests that such patients are at high risk of aortic dissection and aneurysmal rupture at a young age even when the aorta is small. ${ }^{2,3}$ We report

From the Departments of Cardiothoracic Surgery, ${ }^{\mathrm{a}}$ Radiology, ${ }^{\mathrm{b}}$ Cardiolo$\mathrm{gy}^{\mathrm{c}}{ }^{\mathrm{a}}$ and Pathology, ${ }^{\mathrm{e}}$ Stanford University Medical Center, Stanford, Calif; and the Department of Pathology, ${ }^{\mathrm{d}}$ University of Washington, Seattle, Wash.

Received for publication Feb 23, 2007; accepted for publication March 8, 2007.

Address for reprints: R. Scott Mitchell, MD, Stanford University School of Medicine, Department of Cardiothoracic Surgery, Falk Cardiovascular Research Center, Stanford, CA 94305-5407 (E-mail: rsmitch@stanford.edu).

J Thorac Cardiovasc Surg 2007;134:242-3

$0022-5223 / \$ 32.00$

Copyright $\odot 2007$ by The American Association for Thoracic Surgery doi:10.1016/j.jtcvs.2007.03.004

\section{Clinical Summary}

A 23-year-old woman with previous ligation of a patent ductus arteriosus and correction of a cleft palate underwent open repair of a 6-cm infrarenal abdominal aortic aneurysm at an outside hospital. The patient's thoracic aorta was not dilated $(<4 \mathrm{~cm})$. Her physical features included hypertelorism, low-set ears, pectus excavatum, joint laxity, arachnodactyly, and velvety skin texture. Five days postoperatively, she was transferred after an acute Stanford type B aortic dissection developed without signs of organ malperfusion. The dissection originated distal to the left subclavian artery and extended to the infrarenal aortic graft. Computed tomographic angiography showed the maximal diameter of the descending aorta to be $41 \mathrm{~mm}$ at the level of the right pulmonary artery (Figure 1, A). Despite aggressive antihypertensive management, the patient continued to experience intermittent, severe upper back pain. After 7 days, repeat imaging showed that the aorta had enlarged substantially to $45 \mathrm{~mm}$ $(\sim 10 \%$ growth rate) (Figure $1, B)$.

Surgical repair of the aortic dissection was performed through a left thoracotomy with cardiopulmonary bypass and circulatory arrest $\left(17^{\circ} \mathrm{C}\right)$. The descending thoracic aorta, which was severely inflamed, was replaced from the subclavian artery to the diaphrag-

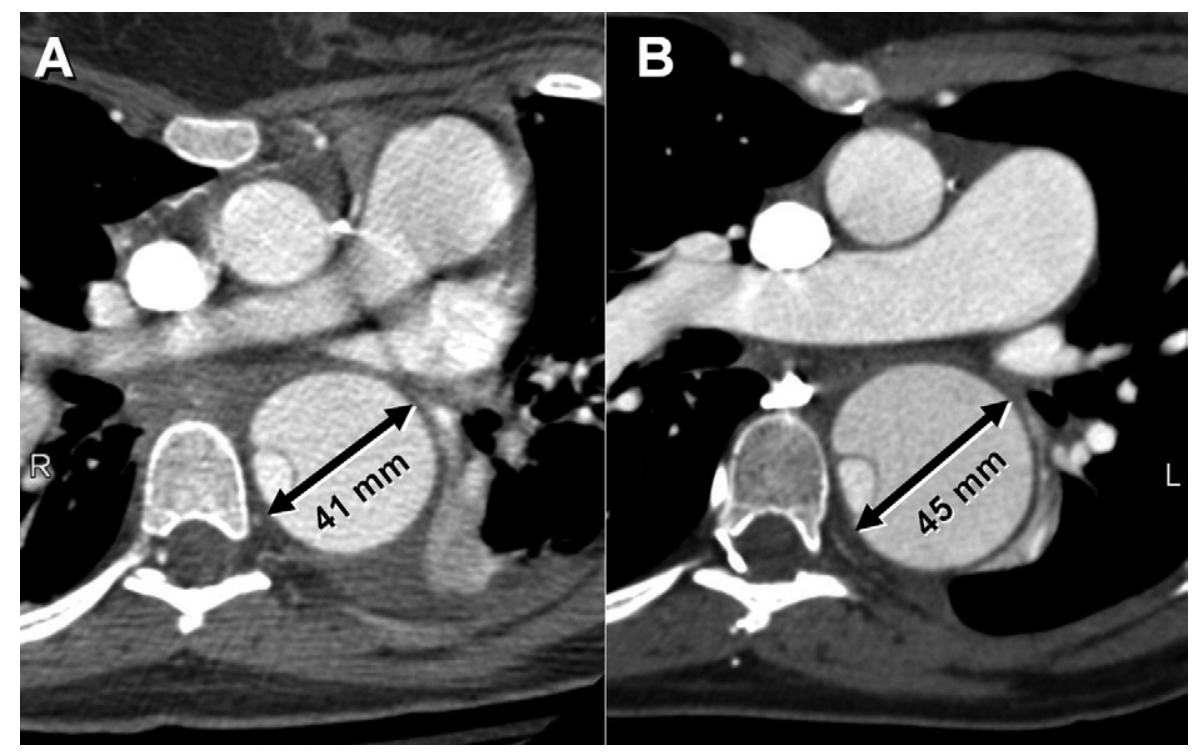

Figure 1. Transverse computed tomographic images at the level of the pulmonary artery demonstrate rapid expansion of the descending aortic aneurysm from $41 \mathrm{~mm}$ (A) to $45 \mathrm{~mm}$ (B) after 7 days. 

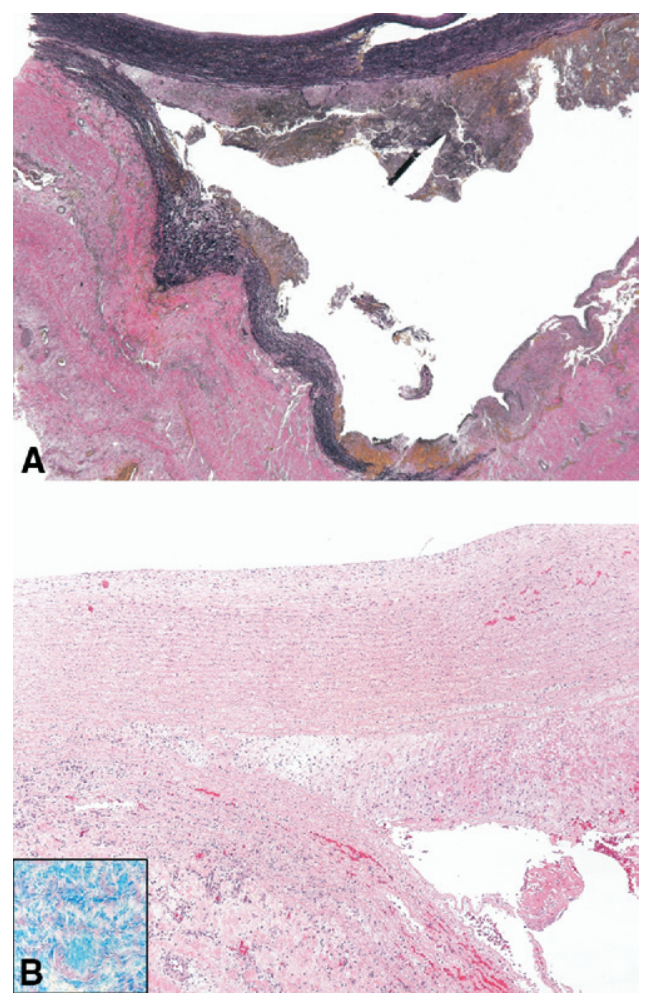

Figure 2. A, Low-power magnification of aortic segment showing the dissection plane in the medial layer. The periadventitial tissue layer is thickened by granulation tissue and fibrous tissue admixed with chronic inflammatory cells (elastic-van Gieson stain $\times 15)$. B, Dissection along the outer portion of the medial layer. The dissection plane contains thrombus and early granulation tissue (hematoxylin-eosin stain $\times 200$ ). Inset, Colloidal iron stain $(\times 400)$ showing abundant mucopolysaccharide accumulations characteristic of cystic medial degeneration.

matic hiatus with a $20-\mathrm{mm}$ polyethylene terephthalate graft. The patient made a full recovery. A postoperative computed tomographic angiogram revealed that the distal dissected thoracoabdominal aorta remained stable (Figure E1, $A$ and $B$ ).

Histologic examination of the aortic wall showed medial dissection with areas of granulation tissue organization (Figure 2,A). The medial layer showed severe cystic medial degenerative changes (Figure 2, B). The diagnosis of Marfan syndrome (MFS) had been considered but was unlikely, as no mutation in the fibrillin 1 (FBNl) gene was found.

Sequencing of DNA from leukocytes identified a missense mutation (guanine to adenosine at nucleotide 1564; c.1564G $>$ A) in exon 7 of one allele of the TGFBR2 gene. This specific mutation, previously not associated with LDS, resulted in substitution of aspartic acid by asparagine at position 522 (D522N) within the highly conserved kinase signaling domain of the TGFBR2 protein. ${ }^{1-3}$ The patient's clinical features of cleft palate, bifid uvula, and hypertelorism, in addition to the mutation in the TGFBR2 gene, confirmed LDS type I. ${ }^{2}$

\section{Discussion}

This report highlights the malignant nature of the aortopathy found in LDS and underscores the need for careful, frequent, and broad surveillance of the entire aorta and peripheral arterial tree, even in patients with aortic dimensions not normally considered to be aneurysmal.

Recent experimental and clinical investigations have suggested a central role for TGF $\beta$ in the pathogenesis of a number of connective tissue disorders. ${ }^{2,4,5}$ Mutations in TGFBRl, $T G F B R 2$, and FBN1 have been shown to lead to derangements in targeting and sequestration of latency-associated peptide with TGF $\beta$, which ultimately increases TGF $\beta$ activity. ${ }^{2,5}$ Histologically, aortic tissue from patients with LDS and from those with MFS demonstrates loss of elastic fibers with marked disarray in the media. ${ }^{1}$

The natural history of LDS has been summarized by Loeys and colleagues. ${ }^{2}$ The reported median survival is 37 years, and thoracic aortic dissection is the primary cause of death (67\%). The median age at first dissection in patients with LDS is 26.7 years. $^{2}$ Given the likely common pathophysiology involved in MFS and LDS, more aggressive operative thresholds for aortic intervention are prudent in patients with LDS, as they are in patients with MFS. Emerging data suggest that the malignant aortopathy found in LDS may be even more aggressive than that of MFS and that the operative thresholds for aortic replacement should be even more stringent. ${ }^{3}$ As our understanding of the aortopathy of LDS increases, the indications for operative intervention in patients with LDS will likely become more clearly defined. The rapidly progressive nature of aortic disease in this patient with an acute type B dissection is consistent with the reports of Loeys, ${ }^{2}$ Williams, ${ }^{3}$ and their colleagues, and further emphasizes the importance of careful aortic surveillance and need for early surgical treatment.

\section{References}

1. Loeys BL, Chen J, Neptune ER, Judge DP, Podowski M, Holm T, et al. A syndrome of altered cardiovascular, craniofacial, neurocognitive and skeletal development caused by mutations in TGFBR1 or TGFBR2. Nat Genet. 2005;37:275-81.

2. Loeys BL, Schwarze U, Holm T, Callewaert BL, Thomas GH, Pannu H, et al. Aneurysm syndromes caused by mutations in the TGF- $\beta$ receptor. N Engl J Med. 2006;355:788-98.

3. Williams JA, Loeys BL, Nwakanma LU, Dietz HC, Spevak PJ, Patel ND, et al. Early surgical experience with Loeys-Dietz: a new syndrome of aggressive thoracic aortic aneurysm disease. Ann Thorac Surg. 2007; 83:757-63.

4. Kaartinen V, Warburton D. Fibrillin controls TGF- $\beta$ activation. Nat Genet. 2003;33:331-2.

5. Dietz HC, Loeys B, Carta L, Ramirez F. Recent progress towards a molecular understanding of Marfan Syndrome. Am J Med Genet. 2005; 139C:4-9. 




Figure E1. Preoperative and postoperative computed tomographic angiograms: Thin-slab maximum-intensity projection (viewed from the left side) of the thoracoabdominal aorta before $(A)$ and after $(B)$ repair of the descending thoracic aorta with a Dacron graft (arrows in A and B). Note the infrarenal aortic graft from prior surgery (arrowheads.) 